\title{
Broadband magneto-optical absorption by a Rashba spintronic system
}

\author{
W. Xu $\mathrm{u}^{\mathrm{a}}$ \\ Institute of Solid State Physics, Chinese Academy of Sciences, Hefei 230031, China \\ and Department of Theoretical Physics, RSPhysSE, Australian National University, \\ Canberra, Australian Capital Teritory 0200, Australia \\ C. H. Yang \\ Faculty of Mathematics and Physics, Nanjing University of Information Science and Technology, \\ Nanjing 219944, China \\ J. Zhang \\ Department of Physics, Yunnan University, Kunming 650091, China
}

(Received 14 October 2007; accepted 1 November 2007; published online 29 November 2007)

\begin{abstract}
We examine the selection rules for electron-photon interaction in a two-dimensional electron gas (2DEG) in the presence of the Rashba spin-orbit interaction and quantizing magnetic fields. In such a case, new channels open up for electronic transition accompanied by the absorption of photons. As a result, two absorption peaks and a broadband absorption spectrum can be observed for high-mobility samples at relatively high magnetic fields. We find that electronic transitions between the neighboring Landau levels within the same spin orientation are the main channels for magneto-optical absorption in a spin-split 2DEG. (C) 2007 American Institute of Physics.
\end{abstract}

[DOI: $10.1063 / 1.2816241]$

The magneto-optical (MO) measurement has been a powerful tool in investigating semiconductor-based electronic devices. ${ }^{1}$ It is known that in a two-dimensional electron gas (2DEG) in which spin-orbit interaction (SOI) is absent or weak, MO transition occurs mainly between neighboring Landau levels (LLs) and within the same spin orientation. ${ }^{2,3}$ When an electron gas is spin translational invariant, although the Zeeman effect splits and shifts the LLs, single absorption peak induced by cyclotron resonance (CR) can be observed when photon frequency $\Omega$ approaches to the cyclotron frequency $\omega_{c}$. For a $2 \mathrm{DEG}$ in the presence of a quantizing magnetic field and of the Rashba SOI (RSOI), ${ }^{4}$ it is found that due to the coupling of the spin orbit to the cyclotron orbit of the system, LL mixing and shifting occur. ${ }^{5}$ Thus, the features of the MO transitions in a spin-split 2DEG may differ from those in a spin-degenerate one. In fact, experimental work on far-infrared MO absorption in InAlAs/InGaAs-based spintronic systems has demonstrated that in the presence of the RSOI, the peak of the CR can be split into two dips under the excitation of pulsed electric or radiation fields. ${ }^{6}$ This feature can be employed to identify the Rashba effect in a spintronic device. ${ }^{6}$ Motivated by these experimental findings, here, we examine the basic selection rules for MO transition in a spin-split 2DEG. We intend studying how the RSOI affects the spectrum of the MO absorption in a $2 \mathrm{DEG}$.

We consider a 2DEG formed in the $x y$ plane and a magnetic field with a strength $B$ is applied along the $z$ axis (growth direction). Including the Zeeman effect and the RSOI, the corresponding Schrödinger equation for an electron can be solved analytically. ${ }^{5}$ When a light field is applied along the $z$ direction of the system and is polarized linearly along the 2D plane (taken along the $x$ direction), the electronic transition rate induced by direct electron-photon interaction can be obtained by Fermi's golden rule. For the case

\footnotetext{
${ }^{a)}$ Electronic mail: wen105@rsphysse.anu.edu.au.
}

of a relatively weak light field so that only the one-photon absorption process is more possible, we have

$$
\begin{aligned}
W_{\lambda^{\prime} \lambda}= & \frac{2 \pi}{\hbar}\left(\frac{e \hbar F_{0}}{\sqrt{2} m{ }^{*} \Omega l_{B}}\right)^{2} \delta_{k_{y}^{\prime}, k_{y}} \delta_{n^{\prime}, n} \delta\left(E_{\lambda^{\prime}}-E_{\lambda}-\hbar \Omega\right) \\
& \times\left(\delta_{N^{\prime}, N-1} I_{N-1}^{s^{\prime} s}+\delta_{N^{\prime}, N+1} I_{N+1}^{s^{\prime} s}\right),
\end{aligned}
$$

with

$$
\begin{aligned}
I_{N \pm 1}^{s^{\prime} s}= & {\left[\sqrt{N+(1 \pm 1) / 2} \cos \theta_{N \pm 1}^{s^{\prime}} \cos \theta_{N}^{s}\right.} \\
& \left.+\sqrt{N-(1 \mp 1) / 2} \sin \theta_{N \pm 1}^{s^{\prime}} \sin \theta_{N}^{s}\right]^{2} .
\end{aligned}
$$

Here, $\lambda=\left(N, k_{y}, n, s\right)$ refers to quantum numbers, $N$ is the LL index, $k_{y}$ is a good quantum number, $s= \pm 1$ refers to the up or down spin state, $n$ is the electronic subband index along the growth direction, $l_{B}=(\hbar / e B)^{1 / 2}, \hbar \Omega_{N}$ $=\sqrt{\left(\hbar \omega_{c}-E_{Z}\right)^{2}+8 N\left(\alpha / l_{B}\right)^{2}}$ with $E_{Z}=g \mu_{B} B$ being the Zeeman energy and $g$ the bare $g$ factor, $\alpha$ is the Rashba parameter which measures the strength of the RSOI, $\theta_{N}^{s}$ $=\theta_{N}+(1-s) \pi / 4$ with $\tan \theta_{N}=\sqrt{8 N}\left(\alpha / l_{B}\right) /\left(\hbar \omega_{c}-E_{Z}+\hbar \Omega_{N}\right)$, $\omega_{c}=e B / m^{*}$ is the cyclotron frequency with $m^{*}$ being the electron effective mass, and $F_{0}$ and $\Omega$ are, respectively, the electric field strength and frequency of the radiation field. Moreover, the energy spectrum of the system is

$$
E_{\lambda}=E_{N, s}+\varepsilon_{n}=N \hbar \omega_{c}+s \hbar \Omega_{N} / 2+\varepsilon_{n},
$$

with $\varepsilon_{n}$ being the electronic subband energy along the growth direction, which indicates that the presence of the RSOI can result in the LL shifting and mixing (see Fig. 1).

With the electronic transition rate, one can employ a semiclassic Boltzmann equation (BE) to study the consequence of a spin-split 2DEG subjected simultaneously to the radiation and magnetic fields. For an electron in the nondegenerate statistics, we have 


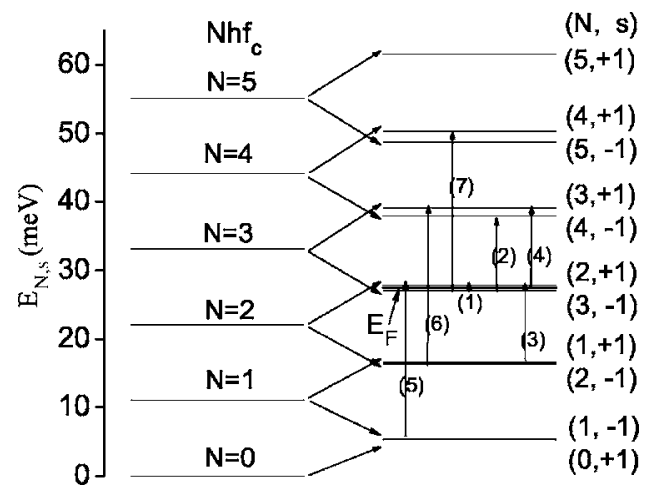

FIG. 1. Spin-split LL structure and MO absorption channels at electron density $n_{e}=5 \times 10^{11} \mathrm{~cm}^{-2}$, magnetic field $B=4 \mathrm{~T}$ (i.e., the filling factor $\nu=5.17$ ), and Rashba parameter $\alpha=1.5 \times 10^{-11} \mathrm{eV} \mathrm{m}$. Here, $E_{F}$ is the Fermi energy, $h f_{c}=\hbar \omega_{c}$, and (i) is an allowed transition channel. Note that (3) and (4) have roughly the same transition energies.

$$
d f_{\lambda}(t) / d t=\sum_{\lambda^{\prime}}\left(F_{\lambda^{\prime} \lambda}-F_{\lambda \lambda^{\prime}}\right),
$$

where $F_{\lambda^{\prime} \lambda}=f_{\lambda^{\prime}}(t)\left[1-f_{\lambda}(t)\right] W_{\lambda^{\prime} \lambda}$ with $f_{\lambda}(t)$ being the electron distribution function at a state $\lambda$. In this study, we apply the usual balance-equation approach to calculate the optical absorption coefficient. ${ }^{7}$ For the first moment, the energybalance equation can be derived by multiplying $\Sigma_{\lambda} E_{\lambda}$ to both sides of the $\mathrm{BE}$, which reads

$$
P(t)=\frac{\hbar \Omega}{2 \pi l_{B \lambda^{\prime}, \lambda}^{2}} \sum_{\lambda^{\prime} \lambda},
$$

where $P(t)=-\left(2 \pi l_{B}^{2}\right)^{-1} d\left[\Sigma_{\lambda} E_{\lambda} f_{\lambda}(t)\right] / d t$ is the electron energy transfer rate. At a steady state, we can use the Fermi-Dirac function as statistical energy-distribution function for an electron, i.e., we can take $f_{\lambda}(t) \simeq f\left(E_{\lambda}\right)$ with $f(x)$ $=\left[1+e^{\left(x-E_{F}\right) / k_{B} T}\right]^{-1}$ and $E_{F}$ being the Fermi energy. Furthermore, we consider a strongly confined 2DEG in which only the lowest electronic subband along the growth direction is occupied by electrons, i.e., we take $n^{\prime}=n=0$ and measure the energy from $\varepsilon_{0}=0$. Thus, the optical absorption coefficient can be calculated through ${ }^{8}$

$$
\alpha_{\mathrm{op}}=\frac{2 P(t)}{\sqrt{\kappa} \epsilon_{0} c F_{0}^{2}}=\alpha_{0} \frac{\hbar \omega_{c}^{2}}{\pi \Omega} \sum_{s^{\prime}, s, N}\left[F_{N+1}^{s^{\prime} s}(\Omega)+F_{N-1}^{s^{\prime} s}(\Omega)\right] .
$$

Here, $\alpha_{0}=e^{2} /\left(\hbar \sqrt{\kappa} \epsilon_{0} c\right), \quad \kappa$ and $\epsilon_{0}$ are, respectively, the dielectric constants of the material and the free space, $c$ is the speed of light in vacuum, and $F_{N \pm 1}^{s^{\prime} s}(\Omega)=f\left(E_{N \pm 1, s^{\prime}}\right)$ $\times\left[1-f\left(E_{N, s}\right)\right] I_{N \pm 1}^{s^{\prime} s} \Gamma /\left[\left(E_{N \pm 1, s^{\prime}}-E_{N, s}-\hbar \Omega\right)^{2}+\Gamma^{2}\right]$, where the broadening of the scattering states has been included, with $\Gamma$ being the broadening of the LLs.

From Eqs. (1a) and (5), we can find the section rules for MO transition via absorption scattering in a spin-split 2DEG. (1) Because the electron-photon scattering does not change the electron wavevector, intra-LL scattering $\left(N=N^{\prime}\right)$ does not contribute to the MO transition. (2) For the case of onephoton absorption, $\mathrm{MO}$ transition occurs between the neighboring LLs (i.e., $\Delta N=1$ ), similar to the case of a spindegenerate 2DEG. (3) In contrast to a spin-degenerate 2DEG in which the MO transition is spin translational invariant, spin-flip transition is allowed for a 2DEG with the RSOI. However, for transition events between the neighboring LLs, the overlap of the electron wavefunctions for different spin Downloaded 14 May 2008 to 150.203.179.187. Redistribution subje

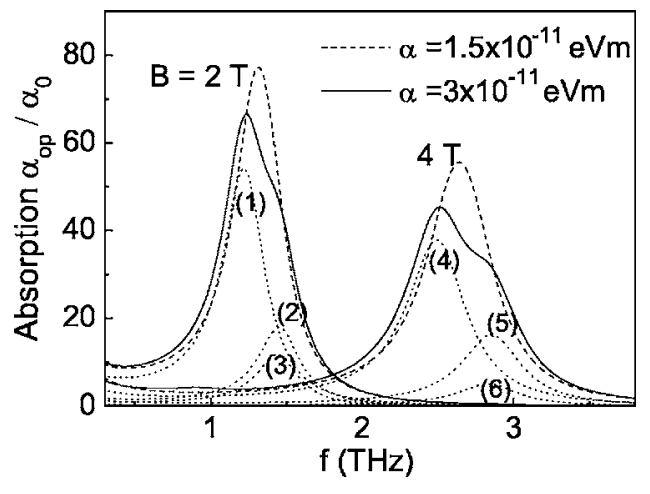

FIG. 2. Optical absorption spectrum at a fixed electron density $n_{e}=5$ $\times 10^{11} \mathrm{~cm}^{-2}$ for different magnetic fields $B$ and Rashba parameters $\alpha$. Here, $f=\Omega / 2 \pi$. For the case of $\alpha=3 \times 10^{-11} \mathrm{eV} \mathrm{m}$ (solid curves), we show contributions from three major transition channels (dotted curves) where (1)-(3) for $B=2 \mathrm{~T}$ and (4)-(6) for $B=4 \mathrm{~T}$.

states (i.e., $s^{\prime} \neq s$ ) is relatively small [Eq. (1b)]. Hence, the major contribution to MO transition via direct electronphoton coupling comes from scattering with the same spin orientation (i.e., $\Delta s=0$ ). Finally, (4) at a low temperature, MO absorption is achieved mainly through transition from occupied states to unoccupied ones. A schematic illustration of the spin-split LL structure and the channels for MO absorption is shown in Fig. 1. We see clearly that in the presence of the RSOI, the LL shifting and mixing can occur and more transition channels (up to seven channels) are allowed according to the selection rules.

In this study, we take the typical sample parameters for an InAlAs/InGaAs-based spintronic device: ${ }^{4}$ the electron density $n_{e} \sim 5 \times 10^{11} \mathrm{~cm}^{-2}$, the Rashba parameter $\alpha \sim 10^{-11} \mathrm{eV} \mathrm{m}$, and the $B=0$ and $T \rightarrow 0$ mobility $\mu_{0}=20 \mathrm{~m}^{2} / \mathrm{V} \mathrm{s}$. We use a simple and well-known result to calculate the LL broadening $\Gamma=\left(2 e \hbar^{2} \omega_{c} / \pi \mu_{0} m^{*}\right)^{1 / 2}$ under the short-range scattering approximation. ${ }^{9}$ The filling of electrons to the LLs and spin states is determined by the condition of electron number conservation, which reads for the filling factor $\nu=2 \pi l_{B}^{2} n_{e}=\Sigma_{N, S} f\left(E_{N, S}\right)$. The results are obtained at a low temperature, i.e., $T \rightarrow 0$.

In Fig. 2, optical absorption coefficient $\alpha_{\mathrm{op}} / \alpha_{0}$ is plotted as a function of radiation frequency at a fixed electron density for different Rashba parameters and magnetic fields. Here, we also show contributions from three major transition channels for the case of $\alpha=3 \times 10^{-11} \mathrm{eV} \mathrm{m}$. At $B=2 \mathrm{~T}$, the filling factor is $\nu=10.34$ and the three major transitions shown in Fig. 2 are (1) for $(6,-) \rightarrow(7,-)$, (2) for $(3,+) \rightarrow(4,+)$, and (3) for $(4,+) \rightarrow(5,+)$. At $B=4 \mathrm{~T}$, the filling factor is $\nu=5.17$ and the three major transitions shown in Fig. 2 are (4) for $(3,-) \rightarrow(4,-)$, (5) for $(1,+) \rightarrow(2,+)$, and (6) for $(2,+) \rightarrow(3,+)$. In the presence of the RSOI, LL mixing and shifting occur so that optical absorption depends strongly on the Rashba parameter $\alpha$ and new channels open up for MO transition (see Fig. 1). In such a case, a broadband absorption spectrum can be seen around the CR frequency. With increasing $\alpha$, a more broadened and damped absorption spectrum can be observed. Because at $T \rightarrow 0$, optical absorption is achieved via exciting electrons from occupied states to unoccupied states, there are three major channels [(1)-(3) at $B=2 \mathrm{~T}$ and (4)-(6) at $B=4 \mathrm{~T}]$ responsible mainly for optical absorption and they all are intra-SO transition events. The numerical results show that the intensity of MO absorpto AIP license or copyright; see http://apl.aip.org/apl/copyright.jsp 


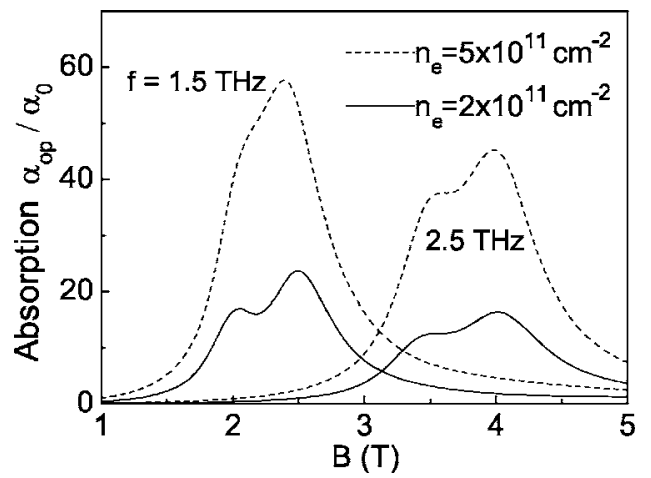

FIG. 3. Optical absorption coefficient as a function of magnetic field at a fixed Rashba parameter $\alpha=3 \times 10^{-11} \mathrm{eV}$ m for different radiation frequencies $f$ and electron densities $n_{e}$, as indicated.

tion via three major transition channels is about two to three orders of magnitude larger than those via spin-flip transitions. We find that the transition energies $E_{4,+}-E_{3,+} \sim E_{5,+}$ $-E_{4,+}$ at $B=2 \mathrm{~T}$ and $E_{2,+}-E_{1,+} \sim E_{3,+}-E_{2,+}$ at $B=4 \mathrm{~T}$. Thus, two absorption peaks can be observed in high-mobility samples (with less LL broadening). Moreover, owing to three major channels for MO transition, a broadband absorption spectrum can be achieved in a $2 \mathrm{DEG}$ with the RSOI. The dependence of the MO absorption on magnetic field is shown in Fig. 3. We see that the two-peak profile of the absorption can be observed more possibly for a low-density sample. This is because it has a less filling factor at a fixed magnetic field. For a high-density sample, more channels for MO transitions can open up so a stronger MO absorption can be observed.

A direct application of the MO spectrum in a spin-split 2DEG is to determine optically the spintronic coefficients (e.g., the Rashba parameter). ${ }^{10}$ At present, the Rashba parameter in InGaAs-based 2DEG systems are commonly measured through magnetotransport (MT) experiments via Shubnikov-de Hass ( $\mathrm{SdH})$ oscillations. ${ }^{4}$ The MT measurement requires Ohmic contact to be made on a sample. The $\mathrm{SdH}$ oscillations are the mixtures of the Zeeman splitting and the Rashba splitting, which are hard to be separated from the measured data. Moreover, the $\mathrm{SdH}$ oscillations can only be observed for relatively low-density samples, otherwise much higher magnetic fields are needed. The MO measurement can overcome these drawbacks in the MT experiments and obtain the spintronic coefficients more easily and accurately. For example, from the positions of two MO absorption peaks, we can determine the occupied (i.e., those $\leqslant N$ ) and unoccupied (i.e., those $\geqslant N$ ) LLs and the Rashba parameter. With these results, the filling factor, $g$ factor, and the total electron density can then be obtained. For an InGaAs-based spintronic system with typical sample parameters, the MO absorption occurs in the terahertz $\left(10^{12} \mathrm{~Hz}\right.$ or terahertz) bandwidth when $B \sim 1 \mathrm{~T}$. This implies that InGaAs-based spintronic systems can be used as broadband terahertz sensors ${ }^{11}$ working at Tesla magnetic fields.

In this work, we have examined the selection rules for MO absorption in a 2DEG in the presence of the Rashba SOI. We find that similar to a spin-degenerate 2DEG, the MO transition in a spin-split 2DEG is achieved mainly via electron-photon scattering within the neighboring LLs and within the same spin orientation. However, due to LL shifting and mixing induced by the coupling of the magnetic field to the spin orbit in a spin-split 2DEG, more channels open up for electronic transition accompanied by the absorption of photons. Thus, two absorption peaks can be observed and a broadband MO absorption spectrum can be achieved. We hope these theoretical predictions can be verified experimentally.

This work was supported by the Australian Research Council, NSF of China (Grant Nos. 10664006 and 90503005), and Knowledge Innovation Program of the Chinese Academy of Sciences.

\footnotetext{
${ }^{1}$ See, e.g., T. S. Moss, Optical Properties of Semiconductors (Butterworths, London, 1959).

${ }^{2}$ Experimentally, see, e.g., C. M. Hu, E. Batke, K. Köehler, and P. Ganser, Phys. Rev. Lett. 76, 1904 (1996).

${ }^{3}$ Theoretically, see, e.g., H. C. A. Oji and A. H. MacDonald, Phys. Rev. B 33, 3810 (1986).

${ }^{4}$ See, e.g., R. Winkler, Spin-Orbit Coupling Effects in $2 D$ Electron and Hole Systems (Springer, Berlin, 2003).

${ }^{5}$ See, e.g., S. Q. Shen, M. Ma, X. C. Xie, and F. C. Zhang, Phys. Rev. Lett. 92, 256603 (2004).

${ }^{6}$ K.-i. Fujii, Y. Morikami, T. Ohyama, S-i. Gozu, and S. Yamada, Physica E (Amsterdam) 12, 432 (2002).

${ }^{7}$ See, e.g., W. Xu, Appl. Phys. Lett. 89, 171107 (2006).

${ }^{8}$ X. L. Lei and S. Y. Liu, J. Phys.: Condens. Matter 12, 4655 (2000).

${ }^{9}$ T. Ando, A. B. Fowler, and F. Stern, Rev. Mod. Phys. 54, 437 (1982).

${ }^{10}$ See, e.g., S. Bandyopadhyay and S. Sarkar, Appl. Phys. Lett. 88, 183108 (2006); S. D. Ganichev, V. V. Bel'kov, G. L. Ivchenko, P. Schneider, S. Giglberger, J Eroms, J. DeBoeck, G. Borghs, W. Wegschneider, D. Weiss, and W. Prettl, Phys. Rev. Lett. 92, 256601 (2004).

${ }^{11}$ For a review, see, e.g., P. H. Siegel, IEEE Trans. Microwave Theory Tech. 50, 910 (2002).
} 